\title{
Anpassung des Mitgliedsbeitrags
}

Der Mitgliedsbeitrag der Arbeitsgemeinschaft Ästhetische Dermatologie und Kosmetologie e. V. liegt seit Jahren konstant bei $40 €$. Anlässlich unserer Mitgliederversammlung in Berlin hat der Vorstand vorgeschlagen, die allgemeine Preis- und Kostenentwicklung zu berücksichtigen und um die Zustimmung zu einer maßvollen Anhebung des Mitgliedsbeitrags um $20 €$ auf nunmehr $60 €$ beginnend ab 2016 gebeten. Dieser Erhöhung wurde einstimmig zugestimmt. Gleichzeitig wurde festgelegt, dass für Assistenten in Weiterbildung der bisherige Beitrag von $40 €$ bestehen bleibt.

Damit soll eine deutliche Intensivierung der Arbeit der ADK e. V. im Hinblick auf die Aus- und Weiterbildung der Mitglieder und insbesondere der Assistenten in der ästhetischen Dermatologie umgesetzt werden. Darüber werden wir im nächsten Heft berichten.
Der Vorstand bittet um Ihr Verständnis für diese schon lange notwendige Entscheidung.

Mit freundlichem Gruß

Dr. Gabriele Feller-Heppt

Schatzmeisterin ADK e. $V$.

Dr. Matthias Herbst

Generalsekretär ADK e.V.

\section{Buchbesprechung "Das diabetische Fußsyndrom. Über die Entität zur Therapie"}

Dieses Buch stellt einen qualitativen Sprung in der Diagnostik und Behandlung des diabetischen Fußsyndroms dar: und zwar im Vergleich zur gesamten bisher veröffentlichten Literatur.

War eigentlich alles zum DFS vermeintlich schon erforscht und breits publiziert, eröffnen die Autoren völlig neue Perspektiven der Betrachtung und Problemlösung. Ausgehend von der Frage: „Warum besteht diese Läsion genau an dieser Stelle?" entfalten sie den Gesamthorizont des aktuellen anatomischen, pathophysiologischen und therapeutischen Wissensbestandes. Das Autorenteam spiegelt dabei die notwendige Kompetenz aus diabetologischer, (fuß-) chirurgischer und wissenschaftlicher Perspektive, die in dieser Konstellation einzigartig ist.

Durch ihre enge Kooperation auch in der alltäglichen Praxis ist ein Lehrbuch „aus einem Guss“ entstanden. Dies erleichtert die Lesbarkeit, die durch reiche Bebilderung noch gesteigert wird. Durch den ständigen Bezug auf die praktischen Konsequenzen der Wundversorgung, der Alternativen externer oder interner, chirurgischer Druckentlastung und der Organisation der Versorgung in der Region empfiehlt sich das Buch für alle Berufs- gruppen, die mit der Behandlung des DFS befasst sind. Es ist damit interdisziplinär, multiprofessionell und transsektoral.

Aus einem reichen Fundus von mehr als 60.000 untersuchten Fällen haben die Autoren prägnanztypische Läsionsorte herausgearbeitet, sog. „Markerläsionen“. An die Lokalisationsbeschreibung schließt sich die Herleitung der jeweiligen Ätiopathogenese und die Definition der Krankheitsentität an. Hieraus ergibt sich dann zwanglos die Ableitung der notwendigen Differenzialtherapien: „Von der Entität zur Therapie“ ist folgerichtig der Untertitel des Buches. Das Kapitel über den Charcot-Fuß folgt dem gleichen Duktus.

Das Entitätenkonzept ist entstanden aus einer jahrelangen Arbeit innerhalb des „Netzwerkes DFS Rheinland“. Diese Erfahrung schildern die Autoren in den letzten Kapiteln zur Organisation der Fußversorgung in der Realdiabetologie. Viele bisher für unlösbar gehaltene Probleme werden hier einer konkreten Lösung zugeführt und mit Behandlungsergebnissen belegt: Die Amputationsrate sinkt.

Erfreulich zudem, dass die Autoren auf die herausragende Bedeutung der
„Das diabetische Fußsyndrom. Über die Entität zur Therapie"

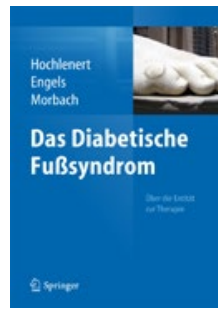

Hochlenert, Engels,

Morbach

Berlin Heidelberg:

Springer-Verlag 2014.

1. Auflage, $2012 \mathrm{~S}$.

ISBN 978-3-662-43943-2

Preis (D): $€ 59,99$

diabetischen Polyneuropathie hinweisen: ohne Leibesinselschwund durch Neuropathie kein DFS. Auch dies findet sich in den üblichen reduktionistischen Lehrbüchern nicht.

Obwohl alle drei Autoren in Schwerpunktpraxis (Engels, Hochlenert) und/ oder Klinik (Engels, Morbach) praktisch tätig sind, imponiert ihr Buch auch durch stupende Beherrschung der nationalen und internationalen Literatur, was dem Gebrauch des Buches als Praxisanleitung keinen Abbruch tut.

Dr. Alexander Risse, Dortmund 